\title{
Disfunções sexuais masculinas em homossexuais: uma revisão integrativa
}

\author{
Male sexual dysfunctions in homosexuals: an integrative review \\ Disfunciones sexuales masculinas en homosexuales: una revisión integradora
}

Recebido: 23/08/2021 | Revisado: 28/08/2021 | Aceito: 30/08/2021 | Publicado: 01/09/2021

\author{
Maria Muniz Coutinho de Oliveira \\ ORCID: https://orcid.org/0000-0002-0142-2104 \\ Faculdade do Vale do Jaguaribe, Brasil \\ E-mail: vitoriamuniz437@gmail.com \\ Kariza Lopes Barreto \\ ORCID: https://orcid.org/ 0000-0002-1406-182X \\ Faculdade do Vale do Jaguaribe, Brasil \\ E-mail: kariza.barreto@fvj.br \\ Roque Ribeiro da Silva Júnior \\ ORCID: https://orcid.org/0000-0001-9517-6823 \\ Universidade do Estado do Rio Grande do Norte, Brasil \\ E-mail: roquejunior@alu.uern.br \\ Glêbia Alexa Cardoso \\ ORCID: https://orcid.org/ 0000-0003-4822-1673 \\ Universidade do Estado do Rio Grande do Norte, Brasil \\ E-mail: glebiacardoso@uern.br
}

\begin{abstract}
Resumo
Introdução: A disfunção sexual é um problema comum a homens e mulheres. Por esse motivo, passou a ser vista como um problema de saúde pública. Contudo, vivemos em uma sociedade onde ainda é restrito falar sobre o comportamento sexual, sobretudo em relação à homossexualidade, definida como atração sexual entre pessoas do mesmo sexo, que está presente desde o início da civilização. Objetivo: foi conhecer a prevalência de disfunções sexuais em indivíduos homossexuais. Metodologia: trata-se de um revisão intergrativa, os dados foram resultantes de uma pesquisa realizada entre março de 2020 a junho de 2021 nas bases de dados PubMed, BVS e PEDro. Para isso, foram incluídos artigos de revisão sistemática e ensaios clínicos randomizados publicados nos últimos 16 anos. Esses relatam sobre disfunção sexual masculina em homossexuais, prevalência, e tratamento das disfunções sexuais. Resultados: Após estudo detalhado, foi analisada a importância de novas pesquisas sobre o tema abordado, visto que a desinformação e a falta de cuidado prevalecem em muitos casos. Consideração Final: portanto, é fundamental a informação e a orientação sobre os cuidados de prevenção e tratamento multidisciplinar, para que tenha uma diminuição das taxas de prevalência, e que com isso esse público passe a ter uma melhor qualidade de vida, e uma vida sexual mais satisfatória e prazerosa.
\end{abstract}

Palavras-chave: Disfunção sexual masculina; Disfunção sexual; Reabilitação sexual; Disfunção sexual em homossexuais.

\begin{abstract}
Introduction: Sexual dysfunction is a common problem for men and women. For this reason, it came to be seen as a public health problem. However, we live in a society where it is still restricted to talk about sexual behavior, especially in relation to homosexuality, defined as sexual attraction between people of the same sex, which has been present since the beginning of civilization. Objective: It was to know the prevalence of sexual dysfunctions in homosexual individuals. Methodology: this is an intergrative review, the data resulting from a survey carried out between March 2020 and June 2021 in the PubMed, BVS and PEDro databases. For this, systematic review articles and randomized clinical trials published in the last 16 years were included. These report on male sexual dysfunction in homosexuals, prevalence, and treatment of sexual dysfunctions. Results: After a detailed study, the importance of further research on the topic addressed was analyzed, as misinformation and lack of care prevail in many cases. Final Consideration: therefore, it is essential to provide information and guidance on preventive care and multidisciplinary treatment, so that there is a decrease in prevalence rates, and that with this this public starts to have a better quality of life, and a sex life more satisfying and pleasurable.
\end{abstract}

Keywords: Male sexual dysfunction; sexual dysfunction; sexual rehabilitation; sexual dysfunction in homosexual men.

\section{Resumen}

Introducción: La disfunción sexual es un problema común para hombres y mujeres. Por esta razón, pasó a ser visto como un problema de salud pública. Sin embargo, vivimos en una sociedad donde todavía se restringe hablar de comportamiento sexual, especialmente en relación a la homosexualidad, definida como atracción sexual entre 
personas del mismo sexo, que ha estado presente desde los inicios de la civilización. Objetivo: conocer la prevalencia de disfunciones sexuales en individuos homosexuales. Metodología: se trata de una revisión integrativa, los datos resultantes de una encuesta realizada entre marzo de 2020 y junio de 2021 en las bases de datos PubMed, BVS y PEDro. Para ello, se incluyeron artículos de revisión sistemática y ensayos clínicos aleatorizados publicados en los últimos 16 años. Estos informan sobre la disfunción sexual masculina en los homosexuales, la prevalencia y el tratamiento de la disfunción sexual. Resultados: Luego de un estudio detallado, se analizó la importancia de profundizar la investigación sobre el tema abordado, ya que en muchos casos prevalece la desinformación y el descuido. Consideración final: por lo tanto, es fundamental brindar información y orientación sobre atención preventiva y tratamiento multidisciplinario, para que haya una disminución en las tasas de prevalencia, y que con esto este público comience a tener una mejor calidad de vida, y una vida sexual más satisfactorio y placentero.

Palabras clave: Disfunción sexual masculina; Disfunción sexual; rehabilitación sexual; Disfunción sexual en homosexuales.

\section{Introdução}

De acordo com Brasil (2013) a Organização Mundial da Saúde reconheceu a função sexual como um indicador de saúde, por esse motivo a disfunção sexual (DS) passou a ser um problema de saúde pública.

A homossexualidade, por sua vez, é definida como a atração sexual entre pessoas do mesmo sexo, estando presente na sociedade desde o início das civilizações. Atualmente, as relações homoafetivas tornaram-se comuns, mesmo diante do preconceito e da falta de suporte, seja ele social, psicológica ou institucional (Kamenov et al., 2017).

Apesar da homossexualidade masculina e as DS serem temas convenientes na literatura científica, a maioria dos homens não dão a devida atenção a essa problemática, sendo, pois, uma questão que necessita de ser mais abordada, visto que alguns estudos relataram episódios de DS em homossexuais do sexo masculino (Grossi, Lucena \& Abdo, 2015).

Dessa forma, é sabido que as pessoas sexualmente ativas estão sujeitas a diversas alterações nas fases da resposta sexual: o desejo, excitação, orgasmo e resolução. Sendo que a DS pode levar à falência em uma ou mais fases desse ciclo (Jacobsen et al., 2019).

Nesse cenário, os principais fatores de risco para DS, característico das populações homossexuais, são aqueles relacionados às questões sociais, como o preconceito e a possibilidade de rejeição. Somam-se a isso o envolvimento com álcool e drogas, violência e pressão sexual, o medo em assumir a própria orientação sexual e homofobia internalizada (Li et al., 2020).

De acordo com a literatura, as DS mais prevalentes, são a disfunção erétil, a aversão a penetração anal, disfunção orgásmica, ejaculação precoce e desejo sexual hipoativo. Porém, essa última não é muito comum (Garrett \& Souza, 2013).

Denomina-se disfunção erétil é a dificuldade de manter uma ereção peniana com intervalo suficiente para o ato sexual satisfatório. A aversão à penetração anal, por sua vez, é o medo da penetração, devido à dor/desconforto causada durante o sexo (Bolat et al.,2017). Por outro lado, a ejaculação precoce ocorre antes ou um minuto após a penetração pois há incapacidade de retardar essa ejaculação. Ademais, a disfunção orgásmica é a dificuldade, demora ou incapacidade de chegar ao orgasmo. Por fim, o desejo sexual hipoativo ocorre quando há estímulos de todos os sentidos, podendo ser eles tátil, visual, auditivo, olfativo e gustativo. (Shendy et al.,2021).

Segundo estudo de Maiorino et al. (2017), foi detectada prevalência de disfunção erétil em extensa amostra realizada através de questionários em homens de todas as regiões do Brasil. O índice identificado compara-se com os de estudos prévios reunidos no Brasil e em outros países. Com isso, é possível confirmar que a disfunção erétil em nosso país é um grave problema de saúde pública, ainda que os homens acometidos por essas disfunções não procurem diagnosticar ou tratar essa disfunção.

Os relatos encontrados na população de nossa pesquisa confirmam as publicações em países com perfis socioeconômicos e culturais distintos do brasileiro. Além disso, há também prevalência dessa anomalia associada a diversas condições clínicas, o que torna essa situação um marcador de saúde, baixa autoestima, relacionamentos conturbados e uma 
menor prática sexual (Wang et al., 2019).

Por outro lado, homens de todas as idades são afetados pela ejaculação precoce. Se tornando a disfunção sexual masculina mais comum, tendo assim, resultados significativamente ruins sobre a qualidade de vida dessas pessoas (Palm et al., 2019).

Ademais, a existência de doenças orgânicas, o uso de medicamentos, de drogas lícitas ou ilícitas são fatores que devem ser investigados em homens que apresentam algum tipo de dificuldade sexual que possa prejudicar o processo de excitação, levando à disfunção (Galati et al., 2014).

Fora isso, além da penetração anal, há inúmeros estímulos para práticas sexuais, não apenas no ânus, mas também no contato com as nádegas, com a região perianal ou anorretal. Neste sentido, é possível haver o contato: pênis-ânus, boca-ânus, dedo-ânus, introdução de objetos sexuais, lubrificantes etc. Independente do estímulo, ele proporciona várias sensações de prazer para os praticantes do ato sexual (Baydoun et al., 2016).

Todavia, devido às inúmeras terminações nervosas no ânus, a dor na penetração anal envolve aspectos fisiológicos e psicológicos. Por isso, é recomendado uso de lubrificantes e posições adequadas para uma melhor penetração. Além do mais, é fundamental trabalhar o psicológico do sujeito que pretende ser penetrado, focando no relaxamento para uma desenvoltura sexual. (Krysiak \& Okopień, 2019).

Neste sentido, se fizermos uma breve análise, ainda existe falta de comunicação e de pesquisa mais aprofundada. Consequentemente, o assunto torna-se interdito, justificando a vergonha e o constrangimento de falar sobre as necessidades do próprio corpo. Além disso, é ainda evidente a desigualdade humana causada pela homofobia. Logo, a falta de compreensão e de conhecimento, inclusive de muitos homossexuais masculinos ou por alguns profissionais da saúde, torna-se dificultosa a incompreensão sobre as práticas sexuais entre homens. (Muin et al., 2018).

Portanto, ainda é restrito falar sobre o comportamento sexual homossexual devido às questões expostas acima e à escassez de estudos aprofundados relacionados a essa temática. Além disso, a maior parte das pesquisas que investigam o comportamento sexual têm geralmente os indivíduos heterossexuais como o principal foco, o que acaba enfatizando ainda mais o tabu e o preconceito, quando é preciso falar sobre as relações sexuais que ocorrem entre pessoas do mesmo sexo. (Silva, 2018).

O objetivo do presente trabalho foi conhecer a prevalência de disfunções sexuais em indivíduos homossexuais, através de revisão de literatura integrativa.

\section{Metodologia}

Neste sentido, esta pesquisa consiste em uma revisão de literatura integrativa. Esta, por sua vez, é compreendida como um método que promove a síntese de conhecimento e a integração da finalidade de resultados de estudos significativos na prática. A revisão integrativa, portanto, é o mais amplo avanço metodológico referente às revisões, permitindo a introdução de estudos experimentais e não-experimentais para uma percepção completa do caso estudado. Além do mais, correlaciona dados da literatura conceitual e da empírica, bem como engloba um vasto conjunto de propósitos, (Souza; Silva; Carvalho, 2010). Diante do exposto, foi realizada uma investigação bibliográfica sobre as disfunções sexuais masculinas em homossexuais, com enfoque na prevalência.

O levantamento de dados foi realizado entre os meses de março de 2020 a junho de 2021, nas plataformas de pesquisa Physiotherapy Evidence Database (PEDro), Biblioteca Virtual em Saúde (BVS) e Medline via Pubmed. Foram selecionados os descritores em ciências da saúde e medical subject headings (DeCs/MeSh): DeCS: disfunção sexual masculina, disfunção erétil, disfunção sexual, reabilitação. MeSh: sexual, male sexual dysfunctions, homossexuais, sexual and gender minorities. Foi utilizado o operador booleano do tipo AND. Com isso as combinações ficaram da seguinte forma na Pubmed: male sexual 
dysfunctions ANDhomossexuais sexuais AND gender minorities, PEDro: erectile dysfunction, sexual dysfunction e BVS: male sexual dysfunctions ANDhomossexuais sexual AND gender minorities, sexual dysfunctions AND gays, conforme o Quadro 1 abaixo.

Quadro 1- Total de artigos por combinações nas bases de dados.

\begin{tabular}{|l|l|c|}
\hline \multicolumn{1}{|c|}{ Bases de Dados } & \multicolumn{1}{|c|}{ Combinações } & \multicolumn{1}{|c|}{ Total } \\
\hline PubMed & $\begin{array}{l}\text { sexual dysfunctions and homossexuais sexual and gender } \\
\text { minorities }\end{array}$ & 274 artigos \\
\hline PEDro & $\begin{array}{l}\text { erectile dysfunction } \\
\text { sexual dysfunction }\end{array}$ & 42 artigos \\
\hline BVS & Male sexual dysfuntions and homossexuais sexual and & 13 artigos \\
& gender minorities & 18 artigos \\
\hline
\end{tabular}

Fonte: Autores (2021).

Assim, foram incluídos apenas os artigos científicos oriundos de ensaios clínicos randomizados, estudo transversal, revisões sistemáticas, dessa forma, foram exclusas os artigos que possuíam duplicidade, trabalhos de conclusão de curso: graduação, especialização, mestrado e doutorado.

A pesquisa foi formulada em duas etapas: A primeira consistiu na leitura e análise do título, do ano de publicação, do resumo, do uso das palavras-chave, do objetivo e o tipo de estudo. A segunda etapa consistiu na ferramenta da qualidade dos estudos. Para isso, observou-se a percepção metodológica, assim como formulários ou ferramentas que foram usufruídos e possíveis restrições do estudo. Com isso, a conclusão do trabalho foi avaliada, considerando, se os termos do estudo obtiveram justificativa nos resultados e quais as advertências do autor. Não houve a aplicação de nenhum formulário durante o procedimento de análise do trabalho.

Nas etapas de análise, apresentação e compreensão dos dados, a princípio, foram demonstrados em quadros e tabelas, aspectos de identificação da metodologia e das evidências analisadas à clareza da literatura. Assim, os principais segmentos dos estudos escolhidos foram estruturados em: número do artigo, autor, ano, tipo de estudo, objetivo e principais considerações. Os artigos foram averiguados acompanhando a concepção da análise temática. Logo, a princípio, conduzimonos à leitura de todo conteúdo. Em seguida, foi priorizada a identificação dos princípios temáticos e, por fim, aferidos os alusivos núcleos. Além disso, os artigos apurados também foram sujeitos à classificação do tipo de metodologia aplicada em cada estudo.

Os conteúdos, por seu turno, foram discutidos e interpretados, considerando a comparação feita sobre eles, a variedade de conhecimento apresentada e a base de informações dos autores. Posteriormente, o texto foi formulado e assinalado, após a leitura do estudo, com o objetivo de coletar a ideia principal do trabalho explorado.

Foram encontrados uma soma de 406 artigos, conforme sua composição numérica nas bases de dados:PubMed ( $\mathrm{n}=$ 274); BVS ( $n=31)$; PEDro ( $n=101)$. Em seguida, foram feitas as exclusões em três fases: a primeira fase contou com os critérios de elegibilidade $(n=265)$ a segunda fase foi leitura avaliativa $(n=115)$, na terceira fase $(n=15)$ por incompatibilidade de conteúdo, analisados através do questionário metodológicos associado a resultados e discussão, resultando em um total de 395 artigos excluídos. Sobejaram um total de 11 artigos com destaque evidente para inclusão. Todavia, devido à inserção dos métodos de inclusão deste estudo, sucedeu ainda a exclusão de 5 artigos, sobrando 6 desses, que foram incluídos na pesquisa, de acordo com a Figura 1 que contém o fluxograma abaixo. 
Figura 1 - Fluxograma.

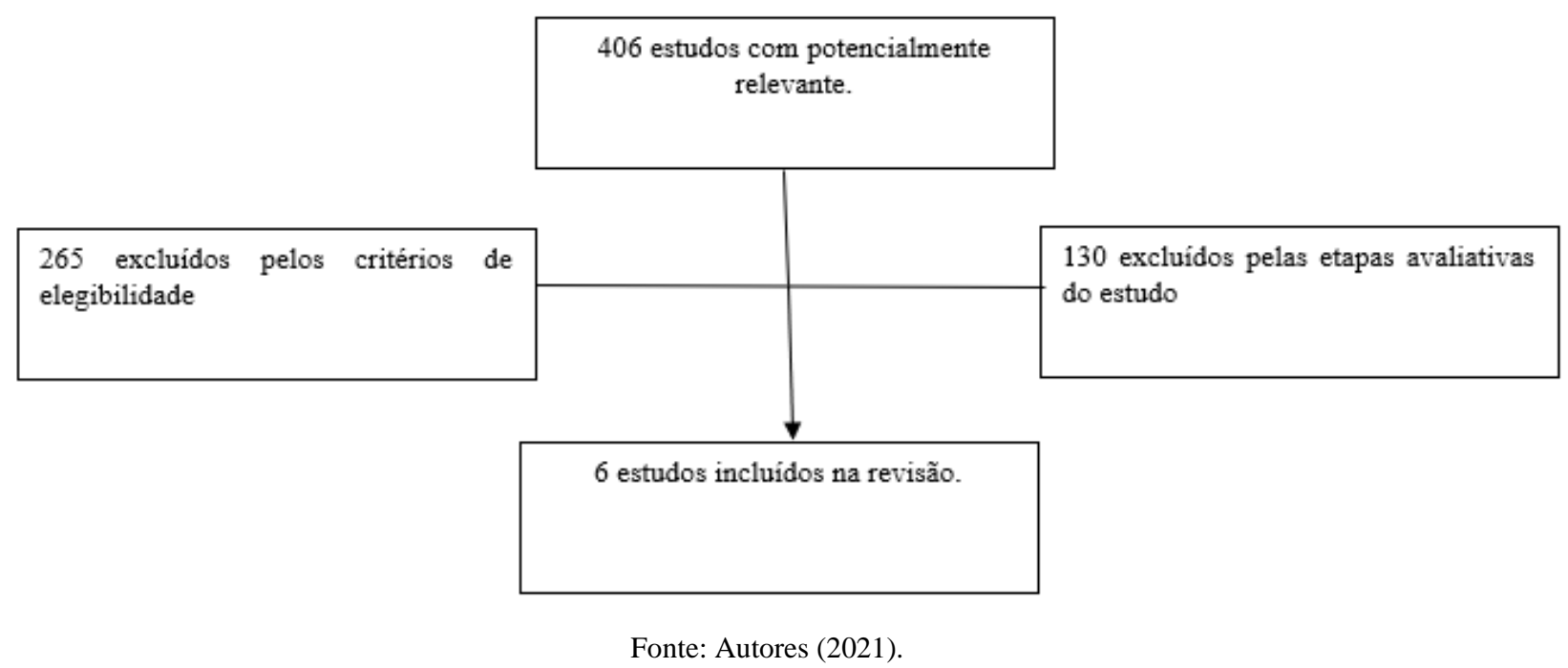

\section{Resultados e Discussão}

Os artigos foram organizados e divididos em tabelas, tal como tópicos. Seguindo uma ordem: número do artigo, autor, ano, tipo de estudo, objetivo, principais considerações.

Quadro 2: Descrição de artigos citados na revisão, dividido em tópicos como: número do artigo, autor, ano, título, tipo de estudo, objetivo, considerações finais.

\section{Artigo 01}

AUTOR (ES):Abdo et al.

ANO DE PUBLICAÇÃO:2006

TíTULO: Disfunção erétil - resultados do estudo da vida sexual do brasileiro.

OBJETIVO GERAL: Estimar a prevalência da disfunção erétil (DE) e fatores de risco associados em amostra da população brasileira.

TIPO DE ESTUDO: Estudo transversal de prevalência

CONSIDERAÇÕES FINAIS:O presente artigo mostra que comparado a outros estudos, é alta a prevalência de disfunção erétil em homens. De modo a ser tratadas com ações terapêuticas, e preventivas para uma melhor qualidade de vida.

\section{Artigo 02}

AUTOR (ES): Francischi et al.

ANO DE PUBLICAÇÃO:2011

TíTULO: Ejaculação precoce: existe terapia eficiente?

OBJETIVO GERAL: Tratamento da ejaculação precoce

TIPO DE ESTUDO: Revisão Sistemática

CONSIDERAÇÕES FINAIS:A ejaculação precoce tem efeitos significativos na qualidade de vida, sendo uma disfunção sexual frequente entre os homens, afetando todas as idades. Existem inúmeros métodos de tratamento sendo utilizados afim de conseguir melhorar a qualidade de vida dessas pessoas. 


\section{Artigo 03}

AUTOR (ES): Garrett \& Souza

ANO DE PUBLICAÇÃO: 2013

TÍTULO: A disfunção sexual em homossexuais masculinos: Potencialidades e desafios

OBJETIVO GERAL: Etiologia, a prevalência, o diagnóstico e o tratamento das disfunções sexuais em homossexuais masculinos.

TIPO DE ESTUDO: Revisão Sistemática

CONSIDERAÇÕES FINAIS: Os principais artigos extraídos dessa revisão mostram a necessidade de mais estudo sobre o tema abordado. A literatura salienta que a terapia sexual concreta pela teoria e a prática, deve-se adaptar-se a esta realidade, de modo a fazer frente às dificuldades desta população específica.

\section{Artigo 04}

AUTOR (ES): Galati et al.

ANO DE PUBLICAÇÃO: 2014

TÍTULO: Sexualidade e qualidade de vida em homens com dificuldades sexuais

OBJETIVO GERAL: Investigar a sexualidade e a qualidade de vida de homens com dificuldades sexuais.

TIPO DE ESTUDO: Ensaio Clínico randomizado

CONSIDERAÇÕES FINAIS:O artigo revelou o relacionamento afetivo-conjugal, qualidade de vida, e o funcionamento sexual, são aspectos importantes nos homens com disfunções sexuais. Entretanto, a necessidade de novas pesquisas é fundamental.

Artigo 05

AUTOR (ES): Grossi, Lucena \& Abdo

ANO DE PUBLICAÇÃO: 2015

TÍTULO: Disfunções sexuais em homossexuais

OBJETIVO GERAL: Prevalência e fatores de risco

TIPO DE ESTUDO: Revisão Sistemática

CONSIDERAÇÕES FINAIS:As disfunções sexuais em homossexuais têm como principais fatores de risco as questões sociais.

\section{Artigo 06}

AUTOR (ES): Baydoun et al.

ANO DE PUBLICAÇÃO: 2016

TíTULO: Fatores biológicos, psicológicos e situacionais da dor durante o intercurso anal em pessoas anoreceptivas Revisão de literatura

OBJETIVO GERAL: Abordar os aspectos biológicos, psicológicos e situacionais que interferem na experiência da dor durante práticas sexuais anais em mulheres e homens anoreceptivos. 
TIPO DE ESTUDO: Revisão Sistemática

CONSIDERAÇÕES FINAIS: Devido a falta de mais estudos sobre essa temática, existe uma enorme demanda, de pessoas à procura de clínicas de proctologia e sexologia, em busca de um tratamento, de prevenção, seja ele medicamentoso, fisioterapêutico, psicoterápicos, elas estão em busca de uma qualidade de vida melhor, e mais prazerosa.

Fonte: Autores (2021).

Contribuindo com o artigo, a pesquisa de Abdo et al. (2006), elaborou um estudo transversal destinado aos heterossexuais, onde desenvolveu o EVSB (estudo da vida sexual do brasileiro), o EVSB foi realizado no Brasil, em 18 cidades, abrangendo todas as regiões do país. Logo em seguida aplicaram um questionário inédito autoresponsivo e anônimo, com 87 itens sobre vários assuntos, como saúde geral, hábitos de vida, comportamento, dificuldades sexuais, o qual os dados seriam subordinados a testes Qui-quadrado e t de Student. Já para avaliar os cálculos de riscos foi aplicado a análise de regressão logística.

No decorrer do estudo foi avaliado que o público de maior número era o da raça negra, poucos da raça amarela, muitos desempregados, e um número bem menor de pessoas com educação superior. Com isso foi comprovado através do estudo traçado, que existe uma alta prevalência de disfunção erétil no brasil, quando quando comparadas a outras pesquisas realizadas dentro e fora do país. Os participantes diagnosticados com DE, apresentam comprometimentos na sua qualidade de vida, com isso a DE se tornou um problema de saúde pública, sendo que a taxa de prevalência e severidade aumenta com o decorrer da idade.

Na pesquisa de Francischi et al. (2011), foi elaborada uma revisão de literaturanos indivíduos heterossexuais, com busca nos estudos que discutissem sobre a ejaculação precoce (EP), de acordo com a pesquisa o autor afirma que a EP é a disfunção que mais acomete os homens de diferentes idades, causando efeitos negativos na qualidade de vida desses indivíduos, abordando também, que os homens que possuem disfunção erétil, também desenvolve a ejaculação precoce.

No estudo de Garrett \& Souza (2013), desenvolveu uma revisão de literaturasobre as DS em homossexuais, que tem como objetivo a etiologia, prevalência, diagnóstico e tratamento das DS em homossexuais masculinos. Em seu estudo, foi relatado que a prevalência das disfunções sexuais masculinas em homossexuais chega a uma porcentagem acima de $90 \%$ pois a maioria dessas pessoas relataram ter, ou já ter tido algum tipo de disfunção sexual ao longo da vida, onde é necessária uma equipe multidisciplinar para trabalhar todas as questões, dúvidas e diagnósticos desses indivíduos, tentando proporcionar uma melhor qualidade de vida para eles.

De acordo com Galati et al. (2014), formulou um ensaio clínico randomizado com público heterossexual, realizado na CIESEX/UNIFESP, foi composta por 10 participantes, que apresentavam queixas sexuais, com idade maior que 40, houve a aplicação de dois questionários respondidos pelos mesmos, para apurar o funcionamento sexual foi aplicado o The GolombokRust Inventory of Sexual Satisfaction(GRISS) e o Short Form Health Survey 36-item (SF-36), avalia a capacidade funcional, dor, saúde geral, aspectos físicos, vitalidade, saúde mental, aspectos sociais e emocionais.Observou-se que todos tiveram algum tipo de DS, com isso a maioria sofreu algum comprometimento sexual devido as disfunções, da mesma forma o autor afirmou que a EP e a DE andam lado a lado de acordo com os resultados.

De acordo com Grossi, Lucena \& Abdo (2015), que teceu um estudo de revisão bibliográfica direcionada aos homossexuais, referiu que a prevalência das disfunções sexuais éconsiderada altas, acima de 50\% dospoucos estudosde prevalência realizados em homossexuais. Em tal caso, o estudo mostrou que as pesquisas confirmam que mais de $90 \%$ dos homens relatamalguma reclamação em relação a função sexual, crendo que o maior fator de risco a DS está relacionado às questões sociais. 
No entanto, Baydoun et al. (2016), desenvolveu uma revisão de literatura sobre a homossexualidade, onde relatou que existe uma alta prevalência de dor/desconforto de moderada a severa nas práticas sexuais anais, no decorrer da leitura do estudo observou-se que os principais motivos de dor/desconforto anal são causados por não trabalhar o psicológico antes e durante o ato sexual, com isso a falta de relaxamento é o fator principal, o tamanho do pênis, a velocidade e profundidade da penetração também são um dos fatores.

Na pesquisa de Silva (2018), realizou uma revisão sistemática direcionada ao público homossexual, para avaliar se o uso do instrumento Male Sexual Function Index (MSFI) em uma versão portuguesa adaptado para homens, a fim de buscar evidências dele, entretanto que não focasse em tipo patológico, de preferência casos não clínicos. Apesar que o estudo cita inúmeros casos de disfunções sexuais masculinas, referindo-se que é comum nos dias de hoje as DS, mas, no entanto, boa parte desse público são desinformados sobre tal assunto, e faz com que isso acabe se agravando com o passar do tempo, e tornando a vida sexual frustrante.

\section{Considerações Finais}

Neste estudo se analisarmos as DS estão presentes na vida da maioria dessas pessoas, confirmando assim, que existe uma alta prevalência de disfunções sexuais de acordo com a literatura, tornando-se um fator de saúde pública. As disfunções sexuais mais comuns e com maior prevalência de acordo com a literatura são a disfunção erétil e a ejaculação precoce. A equipe multidisciplinar de saúde deve melhorar sua forma de abordagem, e de comunicação, de modo que transmita segurança e confiança, para que eles se sintam confortáveis de falar sobre seus problemas sexuais, pois a desinformação e a falta de cuidado são prevalentes, causando a progressão dessas disfunções.

Então é fundamental a informação correta, a orientação sobre os cuidados de prevenção, e assim conseguir tentar prevenir, e tratar uma possível disfunção. Para que as taxas de prevalência diminuam ao longo do tempo, e para que esse público venha a ter uma melhor qualidade de vida, e uma vida sexual satisfatória e prazerosa. Diante disso, a falta de pesquisa sobre essa temática ainda é escassa, novos estudos deverão ser formulados, para que se possa obter mais informações atuais sobre o tema.

\section{Referências}

Abdo, C. H., Oliveira, W. M. J., Scanavino, M. T., Martins, F. G. (2006). Disfunção erétil: resultados do estudo da vida sexual do brasileiro. Revista da Associação Médica Brasileira, 52 (6) 424-429.

Baydoun, M. (2016). Fatores biológicos, psicológicos e situacionais da dor durante o intercurso anal em pessoas anoreceptivas Revisão de literatura Fatores biológicos, psicológicos e situacionais da dor durante o intercurso anal em pessoas anoreceptivas Revisão de literatura.

Bolat, M. S., Akdeniz, E., Asci, R., Erdemir, F., Cinar, O., \& Tomak, L. (2017). Ureterorenoscopy with stenting and its effect on male sexual function: A controlled randomised prospective study. Andrologia, 49(9)

Brasil (2013). Saúde sexual e saúde reprodutiva. Ministério da Saúde. $1^{\text {a }}$ edição.

Francischi, F. B. de, Ayres, D. C., Itao, R. E., Spessoto, L. C. F., Arruda, J. G. F. del, \& Facio Junior, F. N. (2011). Premature ejaculation: is there an efficient therapy? Einstein (São Paulo), 9(4), 545-549.

Galati, M. C. R., Alves Jr., E. de O., Delmaschio, A. C. C., \& Horta, A. L. de M. (2014). Sexualidade e qualidade de vida em homens com dificuldades sexuais. Psico-USF, 19(2), 242-252.

Garrett, A., \& Sousa, M. (2013). Sexual dysfunction in male homosexuals: Strengths and challenges. Analise Psicologica, 31 (1), $103-111$.

Grossi, F. S., Lucena, B. B., \& Abdo, C. H. N. (2015). Disfunções sexuais em homossexuais Sexual dysfunction in gay. Diagn. Trat., $20(1)$, 38-41.

Jacobsen, P., Zhong, W., Nomikos, G., \& Clayton, A. (2019). Paroxetine, but not Vortioxetine, Impairs Sexual Functioning Compared With Placebo in Healthy Adults: A Randomized, Controlled Trial. The journal of sexual medicine, 16(10), 1638-1649.

Kamenov, Z., Fileva, S., Kalinov, K., \& Jannini, E. A. (2017). Evaluation of the efficacy and safety of Tribulus terrestris in male sexual dysfunction-A prospective, randomized, double-blind, placebo-controlled clinical trial. Maturitas, 99, 20-26. 
Research, Society and Development, v. 10, n. 11, e299101119718, 2021

(CC BY 4.0) | ISSN 2525-3409 | DOI: http://dx.doi.org/10.33448/rsd-v10i11.19718

Krysiak, R., \& Okopień, B. (2019). Sexual Functioning in Hyperprolactinemic Patients Treated With Cabergoline or Bromocriptine. American journal of therapeutics, 26(4), e433-e440.

Li, W., Li, S., Lu, P., Chen, H., Zhang, Y., Cao, Y., \& Li, G. (2020). Sexual dysfunction and health condition in Chinese doctor: prevalence and risk factors. Scientific reports, $10(1), 15180$.

Maiorino, M. I., Bellastella, G., Castaldo, F., Petrizzo, M., Giugliano, D., \& Esposito, K. (2017). Sexual function in young women with type 1 diabetes: the METRO study. Journal of endocrinological investigation, 40(2), 169-177.

Muin, D. A., Sheikh Rezaei, S., Tremmel-Scheinost, M., Salama, M., Luger, A., Wolzt, M., Husslein, P. W., \& Bayerle-Eder, M. (2017). Men's sexual response to female partner's intranasal oxytocin administration for hypoactive sexual desire disorder: an open prospective cohort study. Fertility and sterility, 107(3), 781-787.e3.

Palm, P., Zwisler, A. O., Svendsen, J. H., Thygesen, L. C., Giraldi, A., Jensen, K. G., Lindschou, J., Winkel, P., Gluud, C., Steinke, E., \& Berg, S. K. (2019). Sexual rehabilitation for cardiac patients with erectile dysfunction: a randomised clinical trial. Heart (British Cardiac Society), 105(10), 775-782.

Shendy, W. S., Elsoghier, O. M., El Semary, M. M., Ahmed, A. A., Ali, A. F., \& Saber-Khalaf, M. (2021). Effect of low-intensity extracorporeal shock wave therapy on diabetic erectile dysfunction: Randomised control trial. Andrologia, 53(4), e13997.

Silva, A. J. A. (2018) Evidências de validade do male sexual function index em homens de diferentes orientações sexuais. Universidade Federal do Pará.

Soares, C. B., Hoga, L. A., Peduzzi, M., Sangaleti, C., Yonekura, T., Silva, D. R. A. D., Dutra, V. F. D., Oliveira, R. M. P., Zoltowski, A. P. C., Costa, A. B., Teixeira, M. A. P., Koller, S. H. S. H., Grau, D. E. F. E., Evidência, D. E. R. D. E., Souza, M. T. De, Dias, M., Carvalho, R. De Ercole, F. F., Melo, L. S. \& Trevizan, M. A. (2010). Revisão Integrativa versus Revisão Sistemática. Reme: Revista Mineira de Enfermagem, 8(1), 102-106.

Souza, M. T., Silva, M. D., \& Carvalho, R. (2010). Revisão integrativa: o que é e como fazer. einstein (São Paulo), 8(1), 102-106.

Wang, R., Zheng, T., Zhang, T. B., Li, R., Wang, C. L., Yang, F., Jia, D. H., \& Zhang, W. X. (2019). Zhonghua nan ke xue = National journal of andrology, 25(7), 603-607. 